\title{
DUNE Near Detectors with Focus on Cross Sections and Prospects from the Oscillation Perspective
}

\author{
Hongyue Duyang, for the DUNE Collaboration* \\ Department of Physics and Astronomy, \\ University of South Carolina \\ E-mail: duyang@email.sc.edu
}

The DUNE experiment is a proposed next generation long-baseline neutrino oscillation experiment designed to solve mass hierarchy and CP-violation problems by measuring electron neutrino appearance and muon neutrino disappearance in both neutrino and anti-neutrino mode. The DUNE Near Detector, located at Fermilab, will provide crucial constraints on the systematic uncertainties to the oscillation measurements, and in addition, also provide rich physics topics by itself such as neutrino cross-section measurements .

The 19th International Workshop on Neutrinos from Accelerators-NUFACT2017

25-30 September, 2017

Uppsala University, Uppsala, Sweden

${ }^{*}$ Speaker. 


\section{Introduction}

DUNE is the next generation long-baseline neutrino oscillation experiment aiming to solve the remaining problems in neutrino physics, namely the mass hierarchy and CP-violation, by measuring $v_{\mu} / \bar{v}_{\mu}$ to $v_{e} / \bar{v}_{e}$ oscillation [1]. DUNE will use a new neutrino beam proposed at Fermilab, and measure the neutrino spectrum by the near detector (ND) at Fermilab, and the far detector at Sanford Underground Research Facility in South Dakota.

A highly capable near detector will be crucial to DUNE to fulfill its scientific goals. It provide constraints on the systematic uncertainties to the oscillation measurement, such as nuclear effects, neutrino species $\left(v_{\mu} / \bar{v}_{\mu} / v_{e} / \bar{v}_{e}\right)$ and their absolute/relative flux. In addition, the ND will also provide rich physics topics by itself, including precise measurement of neutrino interactions and new physics searches.

There are four near detector design options that have been proposed: Liquid-Argon TPC (LArTPC), the Straw-Tube Tracker (STT, CDR reference design), High-Pressure Argon Gas TPC, and 3D Scintillator Tracker. The collaboration's decision in 2017 is to build a a hybrid detector combining a LAr TPC with one of the other three technologies. All four options are discussed in section 2. Section 3 uses some examples to illustrate the ND strategy of constraining oscillation systematics. Additional ND physics topics, with focus on cross-section measurements, is discussed in section 4 .

\section{DUNE ND Options}

Four options for the DUNE ND have been considered. They will be presented in the following subsections. The DUNE collaboration has reached the agreement that a LAr TPC should be built to provide the same target and detector technology as the far detector. A low-density tracker, using one of the rest three technologies, will be used as a spectrometer for the LAr TPC muons, and also provide low-threshold, high resolution measurement of neutrino interactions to reduce the systematic uncertainties for the oscillation analysis.

\subsection{Liquid Argon TPC}

A Liquid Argon (LAr) TPC will be built as part of the DUNE near detector complex (Figure 1). It has the advantage of using similar technology and same target as the DUNE far detector and making easier the cancellation of detector response and reconstruction uncertainties. In additional, novel technologies are proposed for the ND, including segmented modular design, and pixelized charge readout with high event rate capability. The design has ability to be magnetized. However, the collaboration has made the decision not to magnetize it in the interest of making the detector response similar to the far detector.

\subsection{Straw Tube Tracker}

The Straw Tube Tracker (STT) design was chosen as the reference design for DUNE Conceptual Design Report (CDR). The tracker is composed of straw tubes and radiators and multiple nuclear target. It is surrounded by $4 \pi$ coverage of Electromagnetic-Calorimetors (ECAL) and Muon Detectors (Figure 1). The detector complex is in a dipole magnetic field of $0.4 \mathrm{~T}$ which makes 

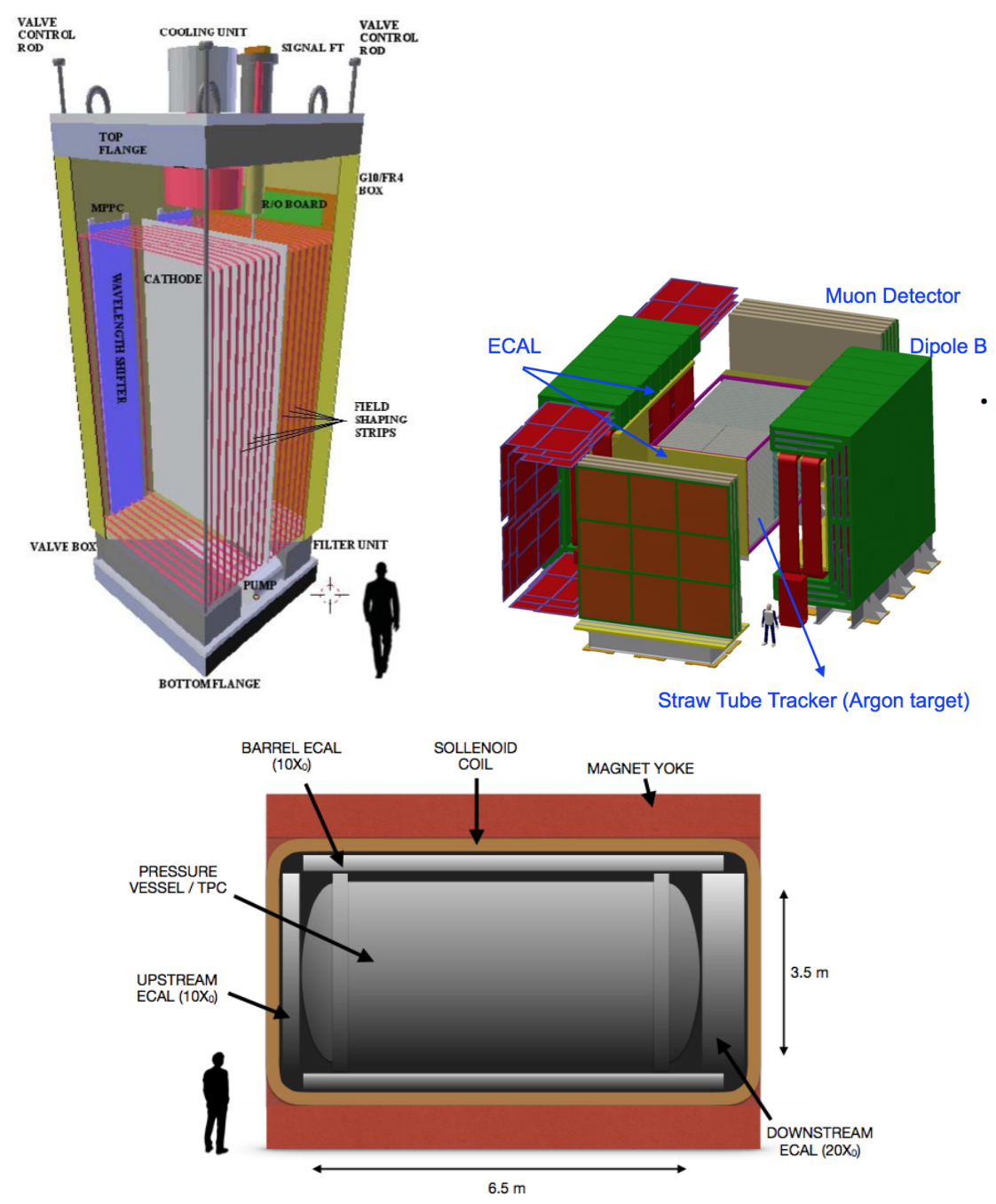

Figure 1: The conceptual pictures showing the DUNE near detector designs, including LAr TPC (top left), Straw-Tube Tracker (top right), and High-Pressure Argon Gass TPC (bottom).

possible the measurement of particle charge and wrong-sign contamination in the (anti)neutrino beam. The proposed nuclear targets include Ar gas which provides 10 times of the far detector statistics, as well as additional target materials such as carbon and calcium to study nuclear effects. The detector is low-density $\left(0.1 \mathrm{~g} / \mathrm{cm}^{3}\right)$, with long radiation length $(6 \mathrm{~m})$, optimized for tracking of charged leptons and hadrons. The FGT detector provides excellent resolution for the electron and muon energy and angle measurement, as well as the high-efficiency background rejection. The detailed performance of FGT is summarized in Table 1.

\subsection{High Pressure Argon Gas TPC}

The High Pressure Argon Gas TPC uses same Ar target as LArTPC with much lower density and thresholds by using Ar gas instead of liquid. It is also compatible with a $0.4 \mathrm{~T}$ magnetic field for the measurement of particle charge. The central part of the detector complex is a large time 
Table 1: STT performance.

\begin{tabular}{|c|c|}
\hline Radiator Mass & 7 tons \\
Other Nuclear Target Mass & $1-2$ tons \\
\hline Vertex Resolution & $0.1 \mathrm{~mm}$ \\
Angular Resolution & $2 \mathrm{mrad}$ \\
Electron Energy Resolution & $6 \% / \sqrt{E}(4 \%$ at $3 \mathrm{GeV})$ \\
Muon Energy Resolution & $3.5 \%$ \\
\hline$v_{\mu} / \bar{v}_{\mu}$ ID & Yes \\
$v_{e} / \bar{v}_{e}$ ID & Yes \\
$\pi^{+} / \pi^{-}$ID & Yes \\
$\pi^{+} / p / K^{+}$ID & Yes \\
\hline $\mathrm{NC} \pi^{0}$ Rejection & $0.1 \%$ \\
$\mathrm{NC} \gamma$ Rejection & $0.2 \%$ \\
$\mathrm{CC} \mu$ Rejection & $0.01 \%$ \\
\hline
\end{tabular}

projection chamber with about 1 tonne of argon pressurized at 10 bar and a pressure vessel that houses the TPC, and $4 \pi$ coverage of electromagnetic sampling calorimeter made of layers of lead and plastic scintillator. Overall it also provide excellent tracking performance and resolution.

\subsection{D Scintillator Tracker}

Scintillator technology has been used by various neutrino experiments (NOvA, T2K, MiNERVA). The DUNE 3DST design takes advantage of this mature technology and our current knowledge from scintillator experiments, and also proposed to use new technology of 3D readout developed by the $\mathrm{T} 2 \mathrm{~K}$ collaboration. This design provides fine-grained, real $3 \mathrm{D}$ reconstruction of neutrino interactions, and fast performance suitable for the high-intensity neutrino beam at DUNE ND.

\section{ND Constraints on Oscillation Systematics}

The most important role of the DUNE near detectors is to constrain the systematic uncertainties for the oscillation measurements. The strategy of uncertainty constraining is under study by the collaboration, and varies between different detector designs. In this section I focus on flux measurements and nuclear effects as example to demonstrate the proposed methods for systematics.

\subsection{Nuclear Effects}

Nuclear effects, including initial state nucleon-nucleon correlations, Fermi motion and finalstate interactions (FSI), are among the most important sources of uncertainties for the neutrino oscillation measurements. Nuclear effects affect neutrino energy reconstruction, and can be different between neutrinos and anti-neutrinos. A low-density tracker can measure both lepton and hadron momenta with low threshold and high precision to calculate transverse kinematic variable (missing- $p_{T}, \phi_{L H}$ which is the angle between the lepton and hadron momentum projections on the transverse plane to the incoming neutrino direction, etc.) which are sensitive to nuclear effects. The measurements of such variables provide handles to constrain nuclear models. In particular, the STT 
design can directly study nuclear effects by providing different nuclear target such as argon, carbon, hydrocarbon, and calcium. Subtraction of interactions on carbon from those on hydrocarbon provides measurements on a free proton target which is very useful for study of neutrino interactions free from nuclear effect. We may also have additional constraint from quasi-elastic (QE), resonance (RES), and coherent $(\mathrm{COH})$ interactions, which will be discussed in the next section.

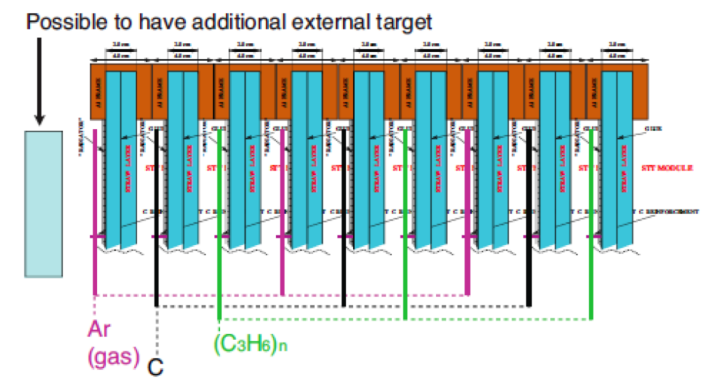

Figure 2: Nuclear targets proposed in the Straw Tube Tracker (STT).

\subsection{Absolute Flux}


Figure 3: Statistical uncertainty of absolute flux measurement expeced in the DUNE ND from neutrinoelectron scattering as function of exposure (left), and distribution of $E \theta^{2}$ of neutrino-electron scattering with different angular resolutions (right). The low-density tracker is expected to have $1-2 \mathrm{mrad}$ angular resolution for electron measurement.

Absolute flux measurement is possible with neutrino-electron scattering: $v_{\mu} e \rightarrow v_{\mu} e$, which is a pure leptonic processes with small but very well known cross-section. The LAr TPC provides enough detector mass for statistics. The most important signature that can be used in selecting neutrino-electrons scattering events is the very small value of $E \theta^{2}$, where $E$ is the energy of the electron and $\theta$ is the angle with respect to the incoming neutrino. A low-density tracker provides high-resolution measurement of electrons which reduces background by tightening $E \theta^{2}$ cut (figure 3). The goal of DUNE ND neutrino-electron scattering measurement is to constrain the absolute flux to the level of $\sim 2 \%$. 


\subsection{Relative Flux}
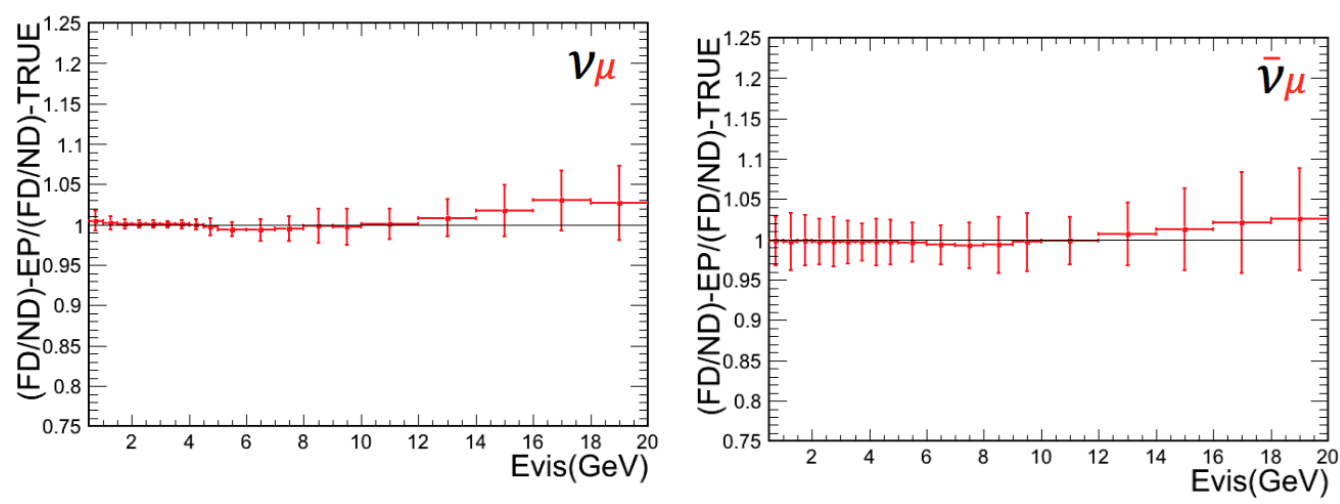

Figure 4: The ratio of expected low- $v$ constrained relative $v_{\mu}$ (left) and $\bar{v}_{\mu}$ (right) flux (as FD/ND ratio) to the default LBNF flux, as function of visible energy.

Relative flux uncertainty can be constrained by using the low- $v\left(v=E_{v}-E_{\mu}\right) v_{\mu}$-CC data in the near detector. At low $v$, the number of events in a given energy bin is proportional to the neutrino flux. The dominant uncertainty to this method is muon energy scale and nuclear effects which affect the measurement of $v$. We expect an FD/ND ratio at $1 / 2 \%$ precision in $0.5 \sim 50 \mathrm{GeV}$ from the low- $v$ method (Figure 4).

\section{Cross-Section Measurement}

A highly capable near detector not only constrains uncertainties to the oscillation measurement, but also has physics topics by itself. This section uses STT as an example to highlight the neutrino cross-section measurement proposed in the DUNE ND.
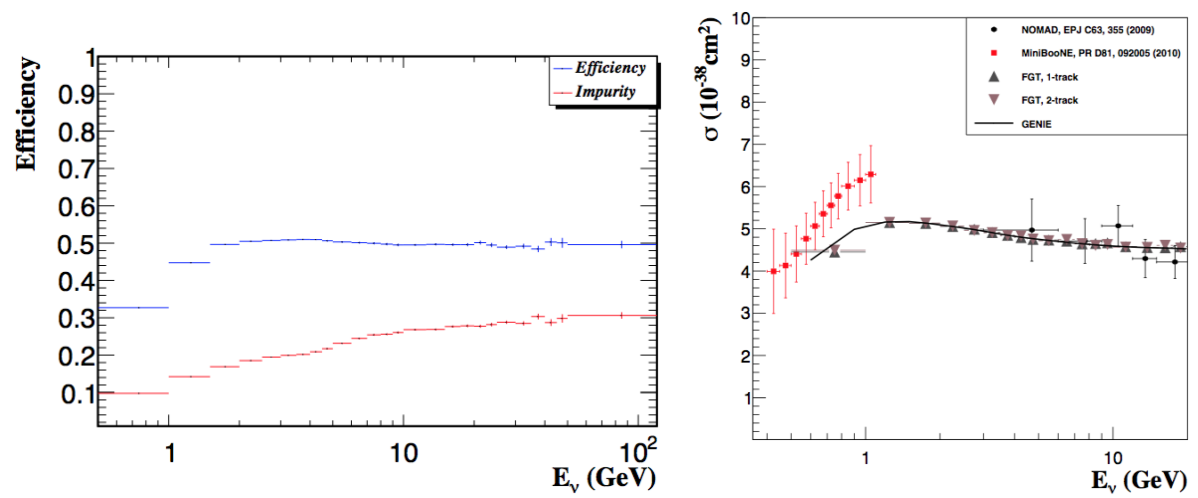

Figure 5: The Quasi-Elastic event selection efficiency and impurity (left) and cross-section study (right) using MC. The cross-section study uses mockdata made from MC, showing result consistent with GENIE prediction, which serves as a validation of the analysis chain.

One example of such study is Quasi-Elastic (QE) interaction, an important channel in DUNE oscillation measurement. Two topologies are possible in the DUNE ND: the first is 2-track topol- 
ogy, where both the outgoing muon and proton are reconstructed; The second is the 1-track topology, where only the muon is reconstructed. Sensitivity study using simulation shows the signal efficiency of QE measurement in STT is $\sim 48 \%$ with $\sim 76 \%$ purity (Figure 5). Besides the crosssection measurement, QE channel can also be used to constrain the nuclear effects, by comparing neutrino energy calculated from muon momentum with the total visible energy .
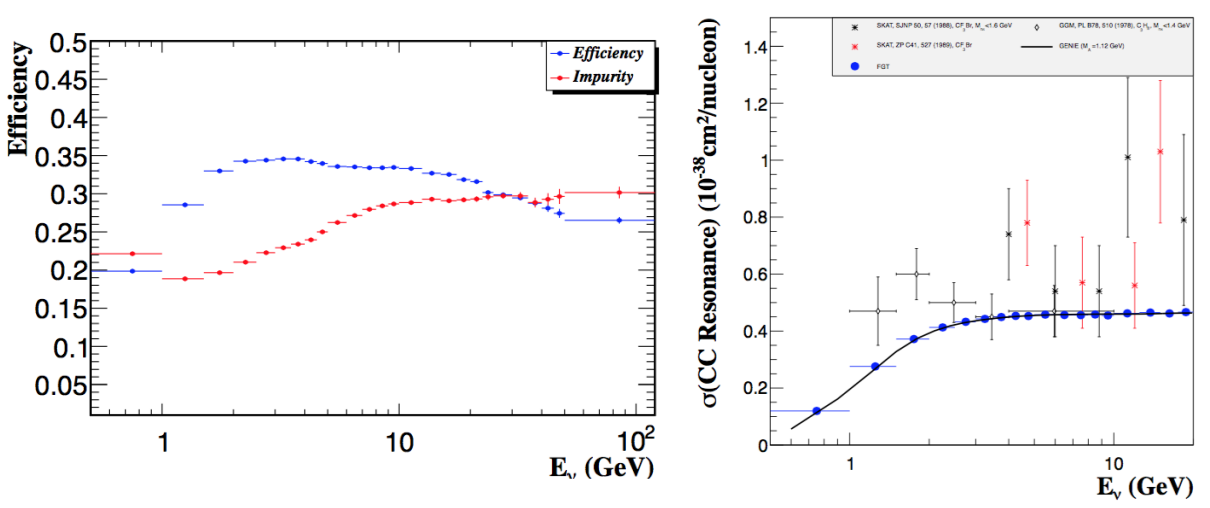

Figure 6: The Resonance event selection efficiency and impurity (left) and cross-section study (right) using MC. The cross-section study uses mockdata made from MC, showing result consistent with GENIE prediction, which serves as a validation of the analysis chain.

Another example of the cross-section measurement is the Resonance (RES) interaction, which will be the most abundant interaction in DUNE detectors. RES also provide an independent handle to constrain nuclear effects by a similar method with QE. Sensitivity study using MC shows that a signal efficiency of $\sim 33 \%$ and purity of $\sim 77 \%$ are expected in STT (Figure 6).

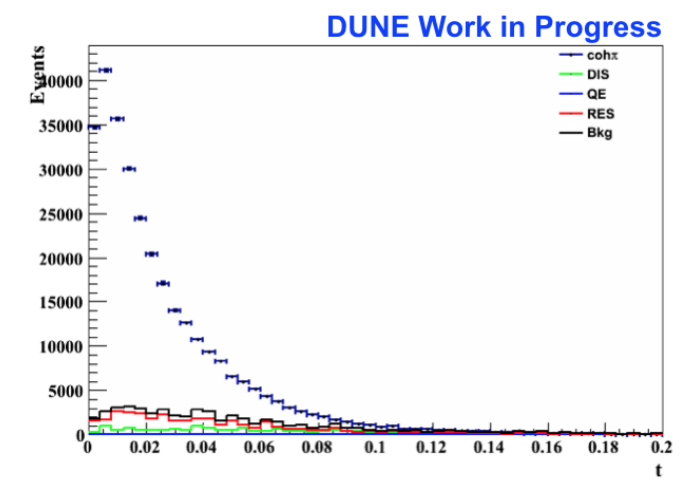

Figure 7: Reconstructed $t$ ( squre of momentum transfer to the nucleus) in STT using simulation with selection cuts applied. Coherent process shows small value of $t$ compared to background.

The last example of cross-section measurements is coherent pion production, which is characterized by very small value of momentum transfer to the nucleus (Figure7). Besides the physics of itself, coherent scattering is the only channel with symmetric topology between neutrino and anti-neutrino interactions $\left(\mu^{-} \pi^{+}\right.$vs $\left.\mu^{+} \pi^{-}\right)$. It is possible to use the coherent channel to measure the wrong-sign neutrino contamination in the neutrino beam. 


\section{Summary}

Four near detector options currently under study by the DUNE collaboration have been discussed. The DUNE collaboration plans to make a final decision of the detector technology by early 2018. We expect the near detector to play an important role in the DUNE experiment, in both the oscillation and cross-sections physics.

\section{References}

[1] R. Acciarri et al. [DUNE Collaboration], arXiv:1601.05471 [physics.ins-det].

[2] S. Mishra, Proceedings of the Workshop on Hadron Structure Functions and Parton Distributions (edited by D. Geesaman et al., World Scientific), 84 (1990). 